\title{
Benign Gastric Ulcer Masquerading as a Submucosal Tumor
}

Benign gastric ulcers typically appear as mucosal defects with various degrees of excavation. We report here an unusual case of a gastric ulcer with extensive mucosal swelling and protrusion, mimicking a submucosal tumor.

A 42-year-old man presented with a three-week history of intermittent melenic stools, without dyspepsia. He was found to be orthostatic and pale, with a hematocrit of $11 \%$ and guaiac-positive stool on rectal examination. Upper endoscopy disclosed a large gastric mass with intact overlying mucosa, arising from the lesser curvature (Figure 1). The distal aspect of the tumor could not be inspected due to an inability to perform retroflexion in this position. A tentative diagnosis of submucosal gastric tumor was made. Shortly after endoscopy, the patient experienced recurrent major bleeding, and required urgent surgical intervention. A distal $50 \%$ gastrectomy was performed, and the pathological examination revealed a $3.5-\mathrm{cm}$ nodular area in the gastric antrum. The center of this lesion contained a $1.3 \mathrm{~cm}$ ulcer, obscured by overhanging mucosal folds. The muscularis mucosae and submucosa adjacent to the ulcer bed had been obliterated and replaced by fibrous tissue (Figure 2). The final diagnosis was benign gastric ulcer.

Benign gastric ulcers can occasionally be misinterpreted as submucosal masses when marked submucosal fibrosis during the healing process leads to elevation and protrusion of the ulcer bed. Endoscopically, a well-circumscribed rounded lesion may be identified projecting into the gastric lumen, thus mimicking a true submucosal gastric tumor, such as leiomyoma, leiomyosarcoma, lipoma, and schwannoma (1). Adachi et al. (2) have described a similar case of a protruding gastric ulcer with a more extensive mucosal defect, initially misleading the authors to suspect a gastric lymphoma. Since the macroscopic appearance of submucosal gastric masses rarely reveals distinguishing characteristics that assist with the endoscopic diagnosis, endoscopic ultrasound (EUS) can be very helpful in evaluating these tumors (3). Okai et al. (4) have recently described the endosonographic features of a patient with gastric ulcer scar simulating a submucosal tumor. EUS might have been helpful in the evaluation of our patient, but would not have altered the management, since the recurrent bleeding required prompt exploratory laparotomy.

This case illustrates the fact that a chronic gastric ulcer can present as a discrete submucosal mass without any evident mucosal defect. Endoscopists should be aware of this uncommon appearance of a gastric ulcer.

K. Mergener', M. K. Washington ${ }^{2}$, T. N. Pappas $^{3}$, A. I. Sharara'

'Dept. of Medicine

${ }^{2}$ Dept. of Pathology

${ }^{3}$ Dept. of Surgery

Duke University, Medical Center, Durham, North Carolina, USA

\section{References}

1. Bandoh T, Isoyama T, Toyoshima H. Submucosal tumors of the stomach: a study of 100 operative cases. Surgery 1993; 113: 498-506.

2. Adachi Y, Mori M, Fujiwara T, et al. Elevated gastric ulcer grossly resembling primary gastric lymphoma. Am J Gastroenterol 1988; 83: 1434-5.

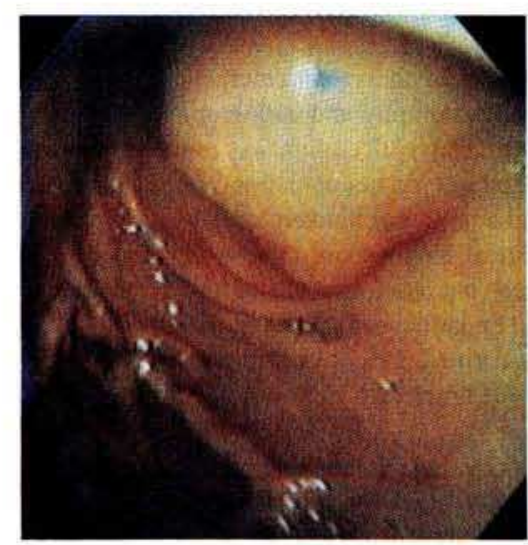

Figure 1: Endoscopic view of the smooth, well-defined protuberant lesion arising from the lesser curvature of the stom ach.

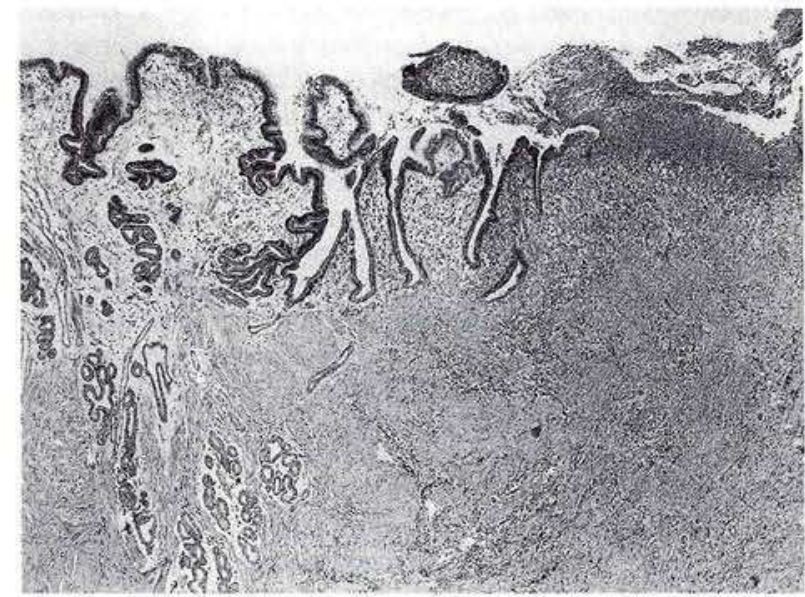

Figure 2: Histological appearance. The ulcer bed is lined by granulation tissue overlying dense fibrous tissue and hyperplastic smooth muscle. Adjacent to the ulcer, the submucosa and muscularis mucosae are obliterated (hematoxylin-eosin, original magnification $\times 40$ ).

3. Rösch T. Endoscopic ultrasonography in upper gastrointestinal submucosal tumors: a literature review. Gastrointest Endosc Clin North Am 1995; 5: 609-14.

4. Okai T, Songuer Y, Watanabe H, et al. Endosonography of a submucosal tumor-like gastric lesion caused by fibrotic ulcer healing. Endoscopy 1995; 27: 128-31.

Corresponding Author

K. Mergener, M.D.

Division of Gastroenterology

Box 3913, Dept. of Medicine

Duke University Medical Center

Durham, NC 27710

USA

Fax: + 1-919-684-4695 